\title{
CONSTRUCCIÓN DEL CONCEPTO MÚLTIPLO COMÚN EN EL DOMINIO DE LOS NÚMEROS NATURALES
}

\author{
Roig, Ana Isabel; Llinares, Salvador y Penalva, María del Carmen \\ Departamento de Innovación y Formación Didáctica. Universidad de Alicante \\ a.roig@ua.es \\ sllinares@ua.es \\ carmina.penalva@ua.es
}

\begin{abstract}
Resumen. El objetivo de esta investigación fue caracterizar el proceso de construcción del concepto de múltiplo común de dos números naturales como parte del esquema de divisibilidad en estudiantes de educación secundaria. A partir de un análisis cualitativo de entrevistas clínicas en las que los estudiantes resolvían y justificaban su proceso de resolución de un problema, identificamos dos momentos cognitivos en el proceso constructivo del concepto de múltiplo común generado durante la resolución del problema. Estos momentos fueron caracterizados teniendo en cuenta cómo los estudiantes generaban casos particulares y coordinaban o no la información procedente de éstos. Esta caracterización proporciona información sobre cómo los estudiantes empiezan a concebir que un número pueda adoptar diferentes papeles en las relaciones multiplicativas del esquema de divisibilidad como parte constituyente del aprendizaje de los números.
\end{abstract}

Palabras clave. Divisibilidad, múltiplo de un número, múltiplo común de dos números, abstracción reflexiva, construcción del conocimiento matemático.

\section{Construction of the common multiple concept in the domain of whole numbers}

Summary. The purpose of this research was to characterize the construction process of common multiple of two whole numbers as a part of divisibility scheme in Secondary School students. From the analysis of clinical interviews with Secondary School students when solving a wordproblem and justifying their decisions, we identify two cognitive moments in the constructive process generated in some cases in the context of problem solving. These cognitive moments were characterized regarding how students generated particular cases and were or were not able to coordinate the information arising from them. The characterization provides us with information about the way in which students begin to conceive that a number can play different roles in the divisibility scheme.

Keywords. Divisibility, multiple of a number, common multiple of two numbers, reflective abstraction, construction of mathematical knowledge.

\section{INTRODUCCIÓN}

Los conceptos de múltiplo, divisor, factor y los procedimientos de descomposición de un número natural en factores primos son fundamentales para la comprensión de la estructura multiplicativa de los números naturales y en particular de la divisibilidad. La estructura multiplicativa puede considerarse como el conjunto de todas las relaciones $a \times b=c$ que también se pueden expresar como $c: b=a$, y complementadas por $a \times b \times c=d$ y $a \times b=d: c$. La comprensión de la estructura multiplicativa incluye experiencia con y conocimiento sobre propiedades tales como conmutatividad, asociatividad, distributividad, equivalencia de $a \times b=c$ y $c: a=b$, y otras propiedades de este tipo (Freundenthal, 1983; Greer, 1994; Harel y Confrey, 1994).

En este contexto, la comprensión de las ideas de divisor, múltiplo y las relaciones «ser divisor de» y «ser múltiplo de» se constituye en un aspecto importante del esquema de divisibilidad en el dominio de los números naturales (Sierra, González, García y González, 1989) y de la relación con su estructura multiplicativa (Vergnaud, 1996; 
Zazkis, 2000). Este hecho, unido a la importancia de la estructura multiplicativa en el desarrollo de otros conceptos matemáticos como la idea de fracción (Empson, Junk, Domínguez y Turner, 2005) o de razón (Ramful y Olive, 2008), ha dado lugar a investigaciones cuyos objetivos han sido caracterizar la comprensión de dichos conceptos y determinar cómo se desarrollan (Campbell y Zazkis, 2002; Zazkis y Campbell, 2006).

La caracterización de la comprensión de las ideas de la teoría de números ha sido investigada desde diferentes perspectivas, poniéndose de manifiesto que dicha comprensión está estrechamente ligada a la construcción de conexiones entre la multiplicación, la división, la factorización y la descomposición en factores primos, de tal forma que establecer estas conexiones permite a los estudiantes analizar las relaciones multiplicativas entre diferentes números (Verschaffel, Greer y Torbeyns, 2006; Zazkis y Campbell, 1996; Zazkis, 1998 y 2008). Por ejemplo, en niveles de secundaria y superiores, se ha estudiado cómo las representaciones decimal y factorial de un mismo número $(235=5 \times 47)$ focalizan la atención de los estudiantes en unas propiedades pero no en otras, permitiéndoles identificar con mayor o menor claridad las relaciones «ser múltiplo de» y «ser divisor de» entre dos números naturales, y por tanto influyendo en la manera en que comprenden las ideas «ser múltiplo de» y «ser divisor de», y sus relaciones (Brown, 2002; Zazkis y Liljedahl, 2004; Zazkis y Gadowsky, 2001).

El desarrollo de las ideas de divisibilidad fue estudiado por Bodí y sus colaboradores (Bodí, 2006; Bodí, Valls y Llinares, 2005 y 2007) identificando niveles de desarrollo del esquema de divisibilidad caracterizados por: a) el uso que los estudiantes hacían de los diferentes modos de representación (representación canónica decimal y representación factorial) y la asunción de la unicidad de la descomposición en factores primos en la identificación de las relaciones «ser múltiplo de» y «ser divisor de»; b) la coordinación de diferentes criterios de divisibilidad que permiten establecer cuándo un número es divisible por otro dado y la asunción de la equivalencia entre « $a$ es divisor de $b »$ y « $b$ es múltiplo de $a » ;$ y (c) el uso de la idea de máximo común divisor y mínimo común múltiplo de dos números en la resolución de situaciones contextualizadas.

Globalmente, estas investigaciones están mostrando que la comprensión de la idea de ser múltiplo puede llegar a ser clave en la comprensión de la equivalencia de las expresiones « $a$ es divisor de $b » \mathrm{y} \ll b$ es múltiplo de $a »$. Esto es debido a la necesidad de que los estudiantes comprendan los diferentes papeles que puede desempeñar un mismo número en estas relaciones. Para indagar en esta cuestión, Brown, Thomas y Tolias (2002) estudiaron la comprensión de la divisibilidad de un grupo de estudiantes para maestros y en particular la idea de múltiplo común. Identificaron tres posibles aproximaciones: la intersección de dos conjuntos de múltiplos, crear múltiplos de uno de los números y dividir por el otro, y utilizar la descomposición en factores primos viendo que una descomposición está incluida en la otra. Aunque estas investigaciones se han centrado en cómo los estudiantes manejaban el algoritmo de cálculo del mínimo común múltiplo de dos números a partir de la descomposición en factores primos (Brown et al., 2002), sus resultados no aportan información sobre la manera en la que los estudiantes llegan a construir el significado de múltiplo común, por lo que es necesario obtener información sobre el proceso de construcción del significado de múltiplo común y de mínimo común múltiplo. Con este artículo pretendemos clarificar algunos aspectos del proceso de construcción que tiene lugar cuando los estudiantes de secundaria resuelven una situación problemática en la que intervienen estos conceptos.

\section{MARCO TEÓRICO}

Las teorías cognitivas que se basan en la abstracción reflexiva de Piaget pueden ayudarnos a explicar cómo se produce la construcción de las ideas matemáticas (Dörfler, 2002; Dubinsky, 1991). Desde una perspectiva piagetiana, el desarrollo de un nuevo concepto matemático es un proceso que conlleva la construcción de estructuras cognitivas a partir de estructuras previamente disponibles. El mecanismo por el cual se lleva a cabo esta construcción es la abstracción reflexiva (Piaget, 2001). Piaget afirma que la abstracción reflexiva es «reflexiva» en dos sentidos complementarios: "Primero, se transpone a un plano superior lo que se toma de un plano inferior (por ejemplo, al conceptualizar una acción). Llamamos a dicha transferencia o proyección un réfléchissement. Segundo, se debe por tanto reconstruir en el nuevo nivel $B$ lo que se ha tomado del nivel previo $A$, o establecer una relación entre los elementos extraídos de A y aquellos todavía situados en B. Esta reorganización que es forzada por la proyección será llamada reflexión (réflexion) en el sentido estricto» (Piaget, 2001, p. 30).

Esta descripción se apoya en la existencia de dos tipos de acciones mentales: la «transferencia a un nivel de cognición superior» (proyección) y la «reconstrucción»(reflexión) en el sentido de establecer relaciones con otras ideas a través de la identificación de regularidades desde un conjunto de registros tomados como ejemplos de los resultados de las acciones cognitivas (Sriraman, 2004). Por ejemplo, un estudiante puede partir de su conocimiento sobre la idea de múltiplo para pensar en un conjunto de múltiplos de un número $a$ y pensar en un conjunto de múltiplos de $b$, pero para llegar a la idea de múltiplo común, es necesario considerar simultáneamente ambos conjuntos de múltiplos y los números que cumplen al mismo tiempo las dos condiciones. Ver un número cumpliendo las dos condiciones al mismo tiempo puede considerarse una «transposición a un plano superior», pues se comienza a pensar sobre estos números más allá de lo que ya se conoce. Una vez reconocida la posibilidad de que un número cumpla las dos condiciones (ser múltiplo de «a» y de «b» a la vez), el estudiante puede iniciar una «reconstrucción» al establecer relaciones con otras ideas (por ejemplo, la manera de identificar esta característica cuando los números están descompuestos en producto de factores primos). En estos momentos es una cuestión pendiente analizar el proceso por el cual los estudiantes llegan a desarrollar estas relaciones que conllevan la construcción del significado de múltiplo común. Esta situación plantea dos cuestiones 
metodológicas: a) cómo dar cuenta de que los estudiantes han «transferido a un plano superior» la idea de múltiplo de un número y caracterizar los mecanismos que llevan a dicha transferencia; y (b) cómo dar cuenta de la «reconstrucción» de la idea de múltiplo en este otro nivel de cognición superior.

Una aproximación teórica para realizar la caracterización de estos mecanismos cognitivos derivados de la idea de la abstracción reflexiva es la propuesta por Simon y sus colaboradores (Simon, Tzur, Heinz y Kinzel, 2004). Estos autores distinguen dos fases del proceso de construcción: la fase de participación y la fase de anticipación. Los resultados de los estudios realizados usando esta aproximación (Tzur, 2003; Tzur, 2004; Tzur, 2007) muestran la solidez del marco en el caso del concepto de fracción unitaria y de la relación de orden inverso entre estas fracciones, proporcionando una caracterización muy detallada del proceso constructivo de estos conceptos a partir de las evidencias empíricas de la distinción entre fases. Es por esta razón por la que escogimos éste como marco teórico para nuestra investigación.

La fase de participación se caracteriza porque,

«el estudiante no tiene todavía la comprensión del elemento (matemático) que es pertinente usar para resolver la situación planteada. En esta fase el estudiante puede realizar diferentes actividades cognitivas y comparar el efecto producido con lo que pretende conseguir, razonando por qué ciertos efectos siguen a su actividad» (Tzur, 2007, p. 277).

Por otra parte, en la fase de anticipación,

«el estudiante puede evocar y utilizar el concepto adecuadamente para resolver una situación problemáti$c a »$ (Tzur, 2007, p. 278).

En la fase de participación, la capacidad del estudiante de razonar sobre la relación entre la actividad mental realizada y los efectos producidos en términos de lo que pretendía conseguir en la resolución del problema (el objetivo) es denominada reflexión sobre la relación actividad-efecto. Tzur (2007) caracteriza este mecanismo de la siguiente manera:

«El mecanismo de reflexión sobre la relación actividadefecto consiste en dos tipos de comparaciones: entre el objetivo del estudiante y los efectos de la actividad, lo que le lleva a realizar (mentalmente) una clasificación de los registros actividad-efecto; y además a realizar una clasificación entre las diferentes situaciones en las que tales registros están involucrados, permitiéndole poder llegar a abstraer una regularidad anticipada y razonada (el concepto)»(Tzur, 2007, p. 276).

El primer tipo de comparación (entre los efectos de sus actividades y el objetivo que pretenden alcanzar) explica la «transposición» a la que hace referencia Piaget. Se produciría cuando el estudiante relaciona las actividades realizadas con sus efectos identificando las que le acercan a su objetivo. Esta perspectiva asume que este proceso se re- pite en las diferentes situaciones de resolución de problemas en las que interviene el concepto matemático, generándose las relaciones con otras ideas (la «reconstrucción» o reorganización que Piaget denominó «reflexión») que permitirán al estudiante anticipar los efectos de sus actividades antes de llevarlas a cabo. La noción de anticipación ha sido usada por Steffe (1994) y Empson y Turner (2006) como clave en la construcción del esquema multiplicativo, y asumimos que la capacidad de anticipar en el contexto de resolución de problemas puede ayudarnos a identificar la construcción del concepto de múltiplo común de dos números entendido como un nuevo concepto matemático en la estructura multiplicativa de los números naturales. Así, desde este marco teórico de referencia y en el ámbito de las investigaciones centradas en la comprensión de los diferentes elementos de la teoría de números, nos planteamos el siguiente objetivo: caracterizar el proceso de construcción del concepto múltiplo común y mínimo común múltiplo de dos números como una manifestación del proceso de abstracción reflexiva de los estudiantes.

Este objetivo se apoya en la hipótesis de que el mecanismo reflexión sobre la relación actividad-efecto, como una particularización de la idea de la abstracción reflexiva, puede aportar información sobre los procesos constructivos que tienen lugar durante la resolución de problemas en fase de participación cuando el estudiante no reconoce el concepto matemático como una herramienta pertinente para su resolución. En este sentido, esta investigación pretende aportar información detallada sobre cómo se genera en estudiantes de secundaria el proceso de abstracción del concepto de múltiplo común y mínimo común múltiplo de dos o más números como un componente de la estructura multiplicativa de los números naturales.

\section{METODOLOGÍA}

\section{Participantes y contexto}

Los participantes en esta investigación fueron 71 estudiantes (38 chicos y 33 chicas) de último curso de Educación Secundaria Obligatoria (15-16 años). Los contenidos de divisibilidad en el currículo de la educación secundaria incluyen las ideas de múltiplo y divisor, números primos y números compuestos, criterios de divisibilidad, relaciones entre «ser múltiplo de» y «ser divisor de», descomposición de un número natural en factores primos y cálculo del máximo común divisor y del mínimo común múltiplo de dos o más números y aplicaciones de la divisibilidad a la resolución de problemas en contexto. Los estudiantes que participaron en esta investigación estaban en la parte final de la educación secundaria, por lo que debían conocer en alguna medida estos contenidos matemáticos.

\section{Instrumentos de recogida de datos: diseño y apli- cación}

Los datos proceden de dos fuentes: 1) la respuesta escrita de los estudiantes a un problema cuya estructura matemá- 
tica se organizaba alrededor de la idea de múltiplo común (Figura 1); y 2) la transcripción de una entrevista clínica posterior a la resolución del problema en la que se pedía a los estudiantes que justificaran las decisiones tomadas y la manera en la que habían resuelto el problema.

El contexto de la resolución del problema permite indagar sobre cómo los estudiantes están comprendiendo los conceptos matemáticos implicados en su resolución, al mismo tiempo que constituye un espacio en el que los estudiantes pueden generar procesos de construcción de los conceptos. Desde esta hipótesis, la entrevista clínica permite que indaguemos sobre los procesos de construcción que tienen lugar en la fase de participación en aquellos casos en los que los estudiantes no han identificado o no han construido previamente la idea de múltiplo común (aunque sean capaces de realizar ejercicios rutinarios de cálculo).

\section{El problema}

Brown y otros (2002) advirtieron que en algunas situaciones problemáticas los estudiantes no reconocían la implicación del mínimo común múltiplo si no se les mencionaba explícitamente la expresión «mínimo común múltiplo». Este hecho parece indicar que el uso de un problema de contexto con dicha estructura implícita puede ser un buen instrumento para estudiar cómo se conocen y cómo se generan los procesos de construcción de los conceptos de múltiplo, múltiplo común de varios números y el mínimo del conjunto de múltiplos comunes, haciendo uso de conocimientos previos sobre la estructura multiplicativa en el dominio de los números naturales.

Teniendo en cuenta esta característica, se diseñó el problema «La pista de baile» (Figura 1), que es una situación estructurada por la idea de múltiplo, múltiplos comunes a varios números y mínimo común múltiplo, y en la que el estudiante tiene que tomar una decisión.

Figura 1

Problema «La pista de baile».

\begin{abstract}
«Un fabricante de baldosas ha donado a la Comisión de Fiestas cierta cantidad de baldosas de $30 \mathrm{~cm}$ de largo y $33 \mathrm{~cm}$ de ancho. La Comisión decide hacer una pista de baile cuadrada en el recinto ferial, pero necesita que les digas:

- Cuál es el lado del menor cuadrado que se puede construir con este tipo de baldosas, sin necesidad de cortarlas, $y$

- Qué otros tamaños podría tener la pista cuadrada para embaldosarla usando sólo baldosas enteras de este tamaño. ¿Por qué?

Resuélvelo y explica qué has hecho para responder a la Comisión».
\end{abstract}

Tzur (2007) indica que para distinguir entre comportamientos de la fase de anticipación y comportamientos de la fase de participación es necesario hacer uso de tareas en las que no se proporcione ningún tipo de indicación. El comporta- miento de los estudiantes ante este tipo de problemas ayuda a diferenciar los casos en los que los estudiantes evocan el concepto matemático que es pertinente para resolver esta situación de aquellos casos en los que los estudiantes no evocan dicho concepto. Es en este segundo caso en el que la resolución del problema se afronta estableciendo conexiones con sus conocimientos previos y construyendo a partir de ellos nuevas estructuras. Por tanto, el estudiante puede generar comportamientos a través de los cuales nosotros como investigadores podamos realizar inferencias sobre la manera en la que se construye el concepto matemático. El problema «La pista de baile» cumple las condiciones exigidas por Tzur (2007) al no incluir ningún tipo de indicación, de forma que si un estudiante no reconoce el concepto de mínimo común múltiplo como instrumento para la resolución del problema (fase de anticipación), se puede esperar que inicie un proceso de construcción del concepto a partir de determinadas acciones cognitivas que le lleven a la abstracción de una idea matemática que le permita resolverlo (fase de participación).

La idea de múltiplo común de dos números interviene en este problema a partir de las cantidades fijas dadas por las dimensiones de las baldosas $(30 \times 33)$ y como cantidades variables tenemos las posibles dimensiones de pista construida con dichas baldosas. La idea de múltiplo común aparece al establecer la relación entre la longitud de los lados de la baldosa y la longitud del lado de la pista cuadrada construida teniendo en cuenta que las baldosas son indivisibles. De esta manera el concepto de mínimo común múltiplo de 30 y 33 permite buscar las dimensiones de la menor pista cuadrada que se puede construir. Es necesario tener en cuenta dos relaciones multiplicativas en la resolución de este problema:

- Con baldosas rectangulares de dimensiones $a \times b$ puede formarse un cuadrado $(l \times l)$ cuyo lado tendrá una longitud múltiplo común de $a$ y $b(l=n \times a=k \times b)$.

- A partir de un cuadrado de lado $c$ se pueden formar cuadrados mayores cuyos lados tendrán longitudes que serán múltiplos de $c(2 c, 3 c, 4 c \ldots) \mathrm{y}$, por tanto, múltiplos comunes de $a$ y $b$.

La consideración de estas relaciones permite aplicar la comprensión de la idea de múltiplo para resolver la situación justificándola adecuadamente. De esta manera, estas características pueden generar procesos de resolución en los que podamos identificar rasgos en los comportamientos de los estudiantes que nos permitan reconocer el proceso de construcción del significado de la idea de múltiplo común durante el proceso de resolución del problema.

\section{La entrevista}

Las 71 entrevistas se realizaron tras la resolución del problema por parte de los estudiantes. Fueron entrevistas semiestructuradas con el objetivo de indagar sobre los procesos de construcción generados durante la resolución del problema. Para ello se contaba con un guión previo en el que se tuvo en cuenta cómo han sido caracterizadas las fases de participación y anticipación en la literatura 
y la información sobre la comprensión de la estructura multiplicativa (Zazkis, 2000). Algunas de las cuestiones iniciales del guión se recogen en la figura 2.

Figura 2

Cuestiones iniciales.

- Explica cómo has resuelto el problema.

- ¿En qué te has fijado para tomar una decisión?

- ¿Cómo sabes cuál es la menor pista que se puede construir?

- ¿Cómo se pueden encontrar otras dimensiones de pistas cuadradas construidas con esas baldosas?

- Si el fabricante está dispuesto a donar una cantidad de baldosas superior a 2.000 pero inferior a 5.000, ¿cuál debe ser la longitud máxima de la pista de baile?

A partir de este guión inicial la entrevista podía derivar hacia aspectos diferentes en función de las respuestas proporcionadas por los estudiantes. Desde este punto de vista, no siempre se realizaban las mismas preguntas a los estudiantes, sino que se adaptaban al proceso de resolución que habían llevado a cabo. Durante la entrevista los estudiantes disponían de sus cuestionarios, ya resueltos, para trabajar sobre ellos y de lápiz y papel por si necesitaban utilizarlos. Todas las entrevistas fueron grabadas en audio y posteriormente transcritas para facilitar su análisis.

\section{Análisis}

En primer lugar, se realizó un análisis descriptivo conjunto de las resoluciones del problema y sus fragmentos de entrevista asociados. Para ello se realizó una narrativa que consistía en una descripción en la que se intentaba unificar la información procedente de ambas fuentes de datos. En una primera etapa de análisis usamos la distinción entre fase de participación y fase de anticipación para categorizar las distintas aproximaciones a la resolución del problema según los conocimientos previos que parecía que los estudiantes ponían en juego (Llinares y Roig, 2008). Esta primera categorización nos permitió, a su vez, advertir la existencia de diferentes grados de sofisticación en los procesos de la fase de participación. Esta situación nos llevó a realizar un segundo análisis centrado sólo en los estudiantes que se consideraba que estaban en la fase de participación del proceso de construcción (es decir, aquellos estudiantes que no reconocían inicialmente la idea de múltiplo común para resolver el problema).

En esta segunda etapa de análisis usamos como referencia el mecanismo reflexión sobre la relación actividadefecto (Simon et al., 2004) y por tanto el foco se centró en las acciones realizadas por los estudiantes y cómo los efectos de estas acciones eran evaluadas por los estudiantes para determinar si les permitían resolver el problema planteado. Con este foco de atención pudimos identificar dos momentos cognitivos diferentes dentro de la fase de participación caracterizados por la manera en la que los estudiantes establecían relaciones entre los diferentes elementos matemáticos a partir del uso de casos particulares siguiendo o no un proceso guiado por un objetivo previamente establecido. Para realizar este análisis seguimos las indicaciones de Clement (2000) en el sentido de que para generar un modelo explicativo del comportamiento de los estudiantes, es necesario seguir un proceso cíclico partiendo de la identificación de ciertos comportamientos y realizando inferencias sobre los posibles mecanismos cognitivos que los apoyan, teniendo en cuenta los resultados de las investigaciones previas y las características del marco teórico de referencia. Estas inferencias son luego comprobadas o rebatidas desde análisis sucesivos.

Siguiendo este proceso identificamos los dos momentos cognitivos a partir de la manera en la que los estudiantes manejaban las dos condiciones del problema, «no se pueden cortar baldosas» $\mathrm{y}$ «la pista tiene que ser cuadrada», para generar un proceso que les permitía identificar o no la idea de múltiplo común de la que previamente no disponía. En este sentido, los momentos cognitivos identificados son manifestaciones del proceso constructivo del mínimo común múltiplo llevado a cabo por los estudiantes durante el proceso de resolución.

\section{RESULTADOS}

De los 71 estudiantes, 43 no resolvieron el problema, o bien mostraron dificultades que les impedían iniciar un proceso de resolución. Algunas de estas dificultades se manifestaban cuando el estudiante consideraba que una de las dos condiciones era imposible (bien la condición «sin cortar baldosas», o bien la condición «pista cuadrada») lo que les llevaba a dar como respuesta que «sería imposible», o indicaban que sería necesario conocer algún dato más. Por otra parte, de los 28 estudiantes restantes, 15 reconocieron la idea de mínimo común múltiplo y la usaron para resolver el problema, mientras que 13 mostraron procesos de generación y coordinación de información relativa a la idea de múltiplo común durante la resolución al no haber reconocido previamente la idea de mínimo común múltiplo o no tener las destrezas procedimentales necesarias. En esta sección describiremos el comportamiento de los estudiantes que sí reconocían la idea de múltiplo común (fase de anticipación) y el comportamiento de los estudiantes que no la reconocían inicialmente (fase de participación) pero desarrollaban procesos matemáticos de generación de información e inferencias que nos permitieron caracterizar el proceso de construcción del concepto de múltiplo común generado durante la resolución del problema.

\section{Fase de anticipación: reconociendo y usando un concepto previamente construido}

El proceso de resolución del estudiante E12 (Figura 3) es un ejemplo prototípico de aquellos que reconocen la idea de mínimo común múltiplo como clave para la resolución del problema y la usan correctamente. Para la resolución del problema, E12 descompone los números 30 y 33 en factores, calcula el mínimo común múltiplo (330) y lo da 
como solución («el lado del menor cuadrado es $330 \mathrm{~cm}$ )) Además, halla el número de baldosas que hará falta en total para cubrir la pista cuadrada (110). También, para buscar otras posibles medidas para el lado de la pista cuadrada considera como soluciones diferentes múltiplos del mínimo común múltiplo anteriormente calculado («todos los múltiplos de $330(660,990 \ldots) »)$. E12 justifica esta decisión explicando que la menor pista cuadrada puede considerarse como «una única baldosa de 330 por $330 \mathrm{~cm}$ » a partir de la cual resultaría fácil construir nuevas pistas cuadradas. Además, considera que las nuevas pistas seguirían cumpliendo la condición «sin cortar baldosas», ya que «al "poner" más baldosas iguales seguiría siendo divisible». Esto pone de manifiesto que la comprensión que ha alcanzado del concepto mínimo común múltiplo le permite buscar nuevos múltiplos comunes de 30 y 33 a partir del mínimo común múltiplo calculando sus múltiplos.

Figura 3

Respuesta de E12 en el cuestionario.

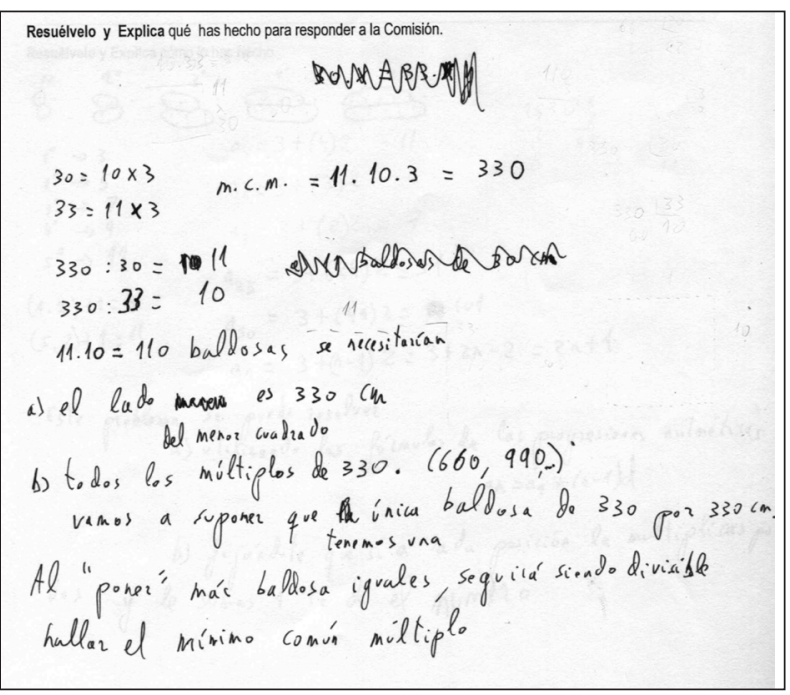

Esta manera de proceder pone de manifiesto una comprensión de la idea de múltiplo común y de mínimo común múltiplo que corresponde a la Fase de Anticipación ya que el estudiante fue capaz de evocar y utilizar el concepto matemático para resolver la situación planteada.

\section{Fase de Participación: Construcción durante el proceso de resolución de problemas}

El comportamiento de los 13 estudiantes que no reconocían la idea de múltiplo común nos permitió identificar cómo se generaban y usaban casos particulares a partir de los cuales buscaban información para poder resolver el problema. La manera en la que los estudiantes coordinaban o no dicha información determina el proceso de construcción del significado de múltiplo común de dos números. Generar casos particulares e intentar coordinar la información a partir de ellos puede ser considerado una manera de buscar relaciones entre su conocimiento previo sobre la estructura multiplicativa y las condiciones del problema planteado. Esta forma de actuar es característica del proceso de construcción que se genera en la fase de participación. Sin embargo, en las 13 respuestas consideradas en la fase de participación se identificaron comportamientos cualitativamente distintos que proporcionan información sobre el proceso constructivo generado durante el proceso de resolución del problema. Tres estudiantes de este grupo realizaron una búsqueda desorganizada que no les permitía avanzar en el proceso de resolución, mientras que los otros 10 mostraron un proceso guiado que ponía de manifiesto la aplicación de una estructura cognitiva para orientar la búsqueda.

El momento cognitivo en el que los estudiantes generan casos particulares para obtener información fue denominado proyección, pues consideramos que se corresponde con la generación de información guiada que permite la «transposición a un plano superior» a la que hace referencia Piaget. El momento cognitivo en el que los estudiantes son capaces de estructurar la búsqueda de nuevos casos particulares fue denominado anticipación local pues consideramos, siguiendo la descripción de la fase de participación de Tzur (2007), que es una manifestación de que una nueva estructura que previamente no existía entra en juego en la resolución de la tarea permitiendo razonar por qué los nuevos casos considerados son cada vez más cercanos a la solución, evidenciándose el mecanismo cognitivo de transposición a un plano superior del conocimiento. Esta anticipación es «local» en el sentido de que la capacidad de anticipar se genera en el contexto de la resolución de la tarea particular. Consideramos que, para que se produzca la transición entre ambos momentos cognitivos, debe haber una reconstrucción de las estructuras cognitivas previas de los estudiantes basadas en el reconocimiento de algunas características de la estructura multiplicativa de la situación, dando lugar a una nueva estructura (el múltiplo común) y por tanto esta transición se ha denominado reflexión en el sentido de Piaget. Las características de estas dos formas de comportarse se describen a continuación.

\section{Generando información sin coordinación}

En algunos casos la generación de casos particulares por parte del estudiante no resultó ser suficiente para la emergencia del significado de múltiplo común. Este hecho pone de manifiesto que es necesario que el estudiante adopte una perspectiva diferente ante el conjunto de datos generado que le permita llegar a construir el significado de múltiplo común. El proceso de resolución llevado a cabo por el estudiante E33 es un ejemplo de este comportamiento en el que no se consigue coordinar la información desde los casos particulares. Este estudiante inicia el proceso de resolución del problema buscando posibles valores para la longitud de los lados con la ayuda de la calculadora. 
88A: Yo... intenté probando, haciendo pruebas en la calculadora.

89E: [...] ¿Qué era lo que probabas?

90A: Espérate que lo lea y te lo digo.

91E: Sí.

92A: [...] Pues ir multiplicando el número de baldosas [...] para que esto y esto al final te diera [...], te diera más o menos... que te diera casi las mismas cifras. [...]

98A: Iba probando en la calculadora.

99E: En la calculadora y no lo encontraste.

100A: Por eso. Creo que para que... porque si quiero hacer algo cuadrado y te dan unas baldosas rectangulares pues no sé, es un poco difícil.

101E: ¿Sí? ¿Pero se podría o no se podría?

102A: [...] Yo creo que no se podría hacer un terreno cuadrado con baldosas rectangulares. Hombre, se podría hacer, como te he dicho, cortando. Pero... yo tampoco sé mucho de esto.

La búsqueda de múltiplos de 30 y 33 se realiza con el objetivo de intentar encontrar medidas iguales, es decir, con el objetivo de cumplir la condición «pista cuadrada». Sin embargo, E33 no supo coordinar la información procedente de estos casos particulares. Esta manera de proceder puede ser considerada como un precursor del hecho de reconocer que para cumplir la condición «sin cortar baldosas» el lado del cuadrado debe ser un múltiplo de las dos longitudes de la baldosa. Así, se inicia una búsqueda entre los múltiplos de 30 y los múltiplos de 33 intentando cumplir la condición «pista cuadrada» de la siguiente manera:

- Colocando cierto número de baldosas a cada lado se tiene Lado $\mathrm{A}_{1}<$ Lado $\mathrm{B}_{1} \rightarrow$ No es un cuadrado.

- Colocando cierto número de baldosa a cada lado se tiene Lado $\mathrm{A}_{2}<$ Lado $\mathrm{B}_{2} \rightarrow$ No es un cuadrado.

Los casos particulares generados y usados por E33 pueden considerarse como un conjunto de registros. El estudiante realiza una serie de acciones (calcular múltiplos) que tienen unos determinados efectos (nunca aparecen dos múltiplos iguales). A partir de los casos particulares, estos estudiantes van creando un conjunto de relaciones de la forma «Lado $\mathrm{A}_{\mathrm{i}}<$ Lado $\mathrm{B}_{\mathrm{j}}$ » al comparar los lados resultantes de la pista. Este proceso crea un conjunto de relaciones que son valoradas por el estudiante en función de la información que pretende recabar. En las líneas 98-102 de la entrevista se pone de manifiesto cómo E33 realiza la comparación entre las actividades realizadas y los efectos producidos generando un conjunto de información que debe manejar. La manera en la que se maneja esta información es clave para el progreso del proceso de construcción.

De esta manera, no encontrar al azar dos medidas que coincidan lleva a E33 a concluir que sería imposible construir la pista requerida por el problema. Esta conclusión puede ser interpretada como una evidencia de que la información procedente de los casos particulares no permite al estudiante reconocer la estructura general de la situación problemática planteada (la idea de múltiplo común).

\section{Realizando una búsqueda guiada de casos particulares}

Los otros 10 estudiantes situados en fase de participación consiguieron coordinar la información procedente de los casos particulares e identificar una regularidad que es usada para resolver el problema. Esta regularidad es el significado de la idea de múltiplo común que empieza a generarse. Esto se manifestaba con la realización de una búsqueda guiada de nuevos casos particulares cada vez más cercanos a la solución.

Un ejemplo de búsqueda guiada lo encontramos en el proceso de resolución de E19. En este caso, el estudiante encuentra un múltiplo común a 30 y 33, el 1320, y parte de él para buscar un tamaño menor. E19 inicia su proceso de resolución calculando múltiplos de 33 y comprobando si son también múltiplos de 30 .

Figura 4

Respuesta de E19 en el cuestionario.

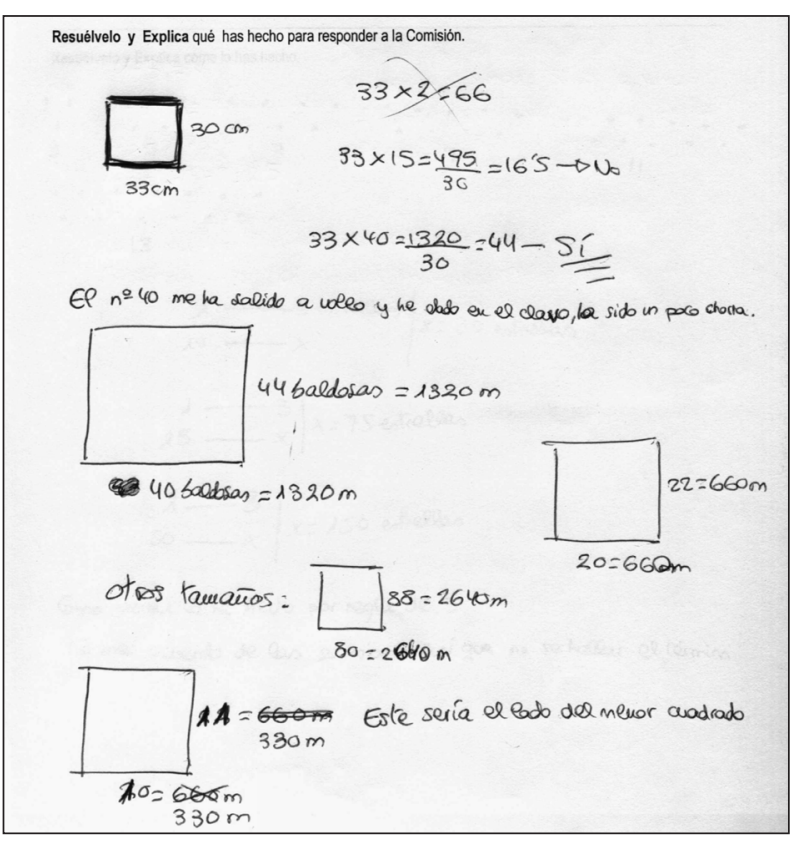

83E: ¿Y qué es lo que estabas calculando? Al poner 33 por 15.

84A: Pues... o sea, de las baldosas el alto que son 33 por 15 baldosas y me da 495 y lo dividí entre 30 que es el otro lado de las baldosas y me daba 16'5 y dije no, porque eso es para partirlo.

La búsqueda del múltiplo común se realiza mediante un proceso de ensayo y error como pone de manifiesto en el cuestionario cuando dice $« \mathrm{El} \mathrm{n}^{\circ} .40$ me ha salido a voleo [de manera arbitraria, sin criterio] y he dado en el clavo, ha sido un poco churra [casualidad, suerte]» (Figura 4).

86A: Y luego pues ya lo dibujé y vi que... entonces tenía que... tenía que dar los mismos metros, ¿no? arriba y 
abajo. Pero luego pensé, bueno pero un divisor pues puede ser 20 y luego pensé pues un divisor de $20 \mathrm{y}$ de 22 , pues puede ser 11 y 10 y entonces al final me quedé con el 11 y el 10 que es el más pequeño.

87E: Muy bien. O sea, que primero calculaste unas posibles dimensiones de la pista que serían según lo que pone aquí 40 baldosas por 44 baldosas.

88A: Sí

89E: Luego fuiste buscando divisores de esos números.

90A: [...] Eso es porque te decía ¿qué otros tamaños podría tener la pista cuadrada?

En su respuesta escrita aparecen otros tamaños de la pista: 88 $\times 80$ baldosas, $22 \times 20$ baldosas y $11 \times 10$ baldosas, indicando que este último sería el que correspondería a la pista de menor lado posible. Según se indica en la entrevista, estos casos los dio como respuesta a la cuestión «¿Qué otros tamaños podría tener la pista cuadrada para embaldosarla usando sólo baldosas enteras de este tamaño?». Estos cálculos se obtienen a partir de la medida inicial $44 \times 40$ multiplicando o dividiendo ambos números por una misma cantidad.

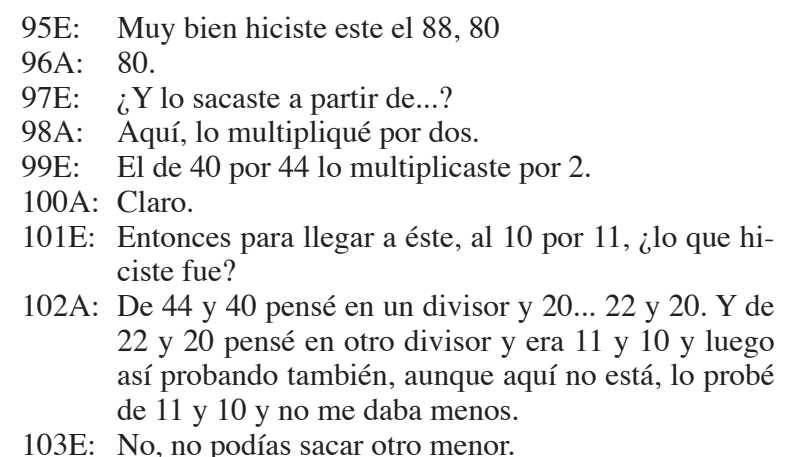

Aunque no hace mención explícita de la regularidad que organiza su búsqueda (la idea de mínimo común múltiplo), parece ser que advierte que al multiplicar obtiene tamaños mayores que el inicial y al dividir obtiene tamaños menores. Esto le lleva a dividir 44 y 40 entre 2, y luego a dividir 22 y 20 entre dos, llegando así al menor lado posible de la pista. Para explicar por qué considera que el caso $11 \times 10$ es el menor, indica que no encontró la forma de seguir dividiendo («lo probé de 11 y 10 y no me daba menos», línea 102), es decir, no encontró un divisor común que permitiese seguir reduciendo el tamaño.

\section{La transición desde la construcción de un conjunto de ca- sos particulares a la búsqueda guiada}

En el transcurso de las entrevistas, algunos de los estudiantes que inicialmente mostraban un comportamiento basado en la simple construcción de un conjunto de casos particulares conseguían coordinar la información y comenzaban a realizar una búsqueda guiada. Esta coordinación de la información es lo que caracteriza el proceso de construcción del conocimiento basado en la abstracción reflexiva. Para ejemplificar esta coordinación utilizamos la entrevista del estudiante E06. Este estudiante no resolvió el problema al completar el cuestionario por desconocer el significado de la palabra baldosa. Durante la entrevista se le explicó el significado de dicha palabra, lo que le permitió empezar la resolución del problema. E06 inició su proceso de resolución con el cálculo de algunos casos particulares en los que «colocaba» el mismo número de baldosas a cada lado de la pista.

120E: Entonces te dan las medidas de los azulejos que tienes.

121A: [...] De ancho sería ésta y esto sería de largo. Una pista de baile cuadrada... Pero cuadrada y la cual el lado... Aquí habría que contar. Si quieren hacer una pista cuadrada, habrá que calcular. Como tenemos ancho 33, nos daría por ejemplo 6. Yo hago mis cálculos.

122E: Sí. Calcula, calcula.

123A: 33 por ejemplo... El número 3 es múltiplo... Esto... Ahora que estoy pensando... No sé cómo calcular esto.

124E: ¿Qué estás intentando hacer?

125A: Intento calcular para que me de un número fijo, para sacar el cuadrado [...] Sería hacer en tabla.

E06 se plantea como objetivo realizar ciertos cálculos para encontrar «un número fijo» (línea 125). El hecho de que se proponga hacer una tabla (línea 125) indica que su idea es organizar los diferentes casos particulares.

131A: Podemos decir que una pista sería una de... [...]. No sé, 6 cuadrados de éstos [refiriéndose a las baldosas]. Poner aquí [horizontal] como 100 da y aquí [vertical] lo mismo. No sé estoy pensando.

132E: Sí, piensa, piensa. Tómate el tiempo que quieras.

133A: [...] Yo haría las cuentas por hacer, como multiplicando por algunos números [...] Aunque todavía puede ser más fácil para algunas personas.

134E: ¿Cómo dirías que sería más fácil?

135A: No sé, si hubiera alguna fórmula para esto. Y si igualmente puede ser que hay, pero no sé [...] Tenemos que hacer una pista igual de grande. Como tenemos un cuadradito aquí de éstos [refiriéndose a una baldosa]... Una pista igual de 300 por ejemplo, 330... Es que eso no es un cuadrado.

No llega a realizar la tabla pero sí empieza a considerar casos en los que va «colocando» diferente número de baldosas. Esta forma de proceder puede ser considerada como un primer paso para realizar una búsqueda guiada. Cuando E06 hace referencia al caso en el que se colocarían 100 baldosas (línea 131), se puede interpretar que E06 está considerando el mismo número de baldosas en ambos lados de la pista. Siguiendo esta estrategia, E06 intenta colocar 10 baldosas a cada lado de la pista y calcula sus dimensiones obteniendo $300 \mathrm{~cm}$ para un lado y $330 \mathrm{~cm}$ para el otro (línea 135). De esta manera E06 empieza a construir un conjunto de registros desde el que obtiene información que le indica que en ninguno de los casos considerados se obtiene un cuadrado. Sin embargo, ante la imposibilidad de construir así una pista cuadrada no se limita a concluir, como sucedía en el caso del estudiante E33, que sería imposible. E06 empieza a coordinar la información que le proporciona este conjunto de casos particulares advirtiendo que, como 33 es mayor que 30, deberá poner menos baldosas en el lado de la pista formado con el lado mayor de la baldosa y más baldosas en el otro lado de la pista. 
136E: Claro, eso no es un cuadrado. Pero la pista tiene que ser cuadrada.

137A: Sería de ancho menos y de largo más cuadrados. Pienso yo, para que quede...

138E: ¿Y no se podría hacer una pista cuadrada?

139A: Sí, sí. Pero quiero decir que de ancho...

140E: ¡Ah, que tendría que haber más!

141A: Sí, como haber más cuadrados [baldosas] de ancho y de largo menos.

La comparación entre las longitudes obtenidas en los casos particulares junto a la consideración de la condición «pista cuadrada», le lleva a advertir que es necesario colocar distinto número de baldosas a cada lado $(33 \cdot a$ $=30 \cdot b$ con $a \neq b$ ) por lo que una de las cantidades debe ser mayor que la otra $(33 \cdot a=30 \cdot b$ con $a<b)$. De esta manera, la información procedente de los casos particulares es coordinada para empezar a ajustar la búsqueda de casos particulares al objetivo pretendido.

145E: Podemos decir que si multiplicamos esto por 10 [...] nos da 330 y si multiplicamos esto nos da 300 ¡No! 300 y $330[\ldots]$ pero como yo pienso que esto tiene que ser más [...] He multiplicado [...] 33 por 10 que nos daría 330

146A: Sí.

147E: Y entonces, como yo pensé que tiene que haber más baldosas de largo que de ancho, multipliqué por un número más que es 11 por 30 , y me dio lo mismo, 330. La pista sería de 10 y 11.

E06 parte de una búsqueda de medidas particulares para los lados de la pista y llega a encontrar el valor 330 como solución cuando afirma «que tiene que haber más baldosas de largo que de ancho» (línea 147) y por eso multiplicó 30 por un número mayor. Aplicar esta relación para ajustar sus cálculos le lleva a pasar de multiplicar 30 por 10 a multiplicar 30 por 11. Esta forma de proceder indica que la búsqueda es guiada en el sentido de que no se realiza de forma arbitraria sino que se aplica considerando una estructura que le permite ir aproximándose a la solución.

De esta manera, las relaciones claves que el estudiante necesita construir durante la resolución del problema para poder usar la idea de múltiplo en esta situación pasa por advertir que es necesario poner distinto número de baldosas a cada lado de la pista para poder construir un cuadrado desde una baldosa rectangular y observar la relación inversa entre el número de baldosas a colocar en cada lado y la longitud del lado de las baldosas. En algunos de los protocolos analizados los estudiantes coordinaban la información desde un análisis cualitativo de la situación. Una descripción detallada de este tipo de comportamiento puede encontrarse en Roig (2008) poniéndose de manifiesto que también en estos casos la clave estaba en la coordinación como punto de apoyo de la búsqueda guiada.

\section{DISCUSIÓN Y CONCLUSIONES}

La distinción teórica entre fase de participación y fase de anticipación, junto al mecanismo reflexión sobre la relación actividad-efecto han sido usados para describir cómo se desarrolla el proceso de construcción del concepto de múltiplo común. Esta descripción nos ha permitido identificar dos momentos en la fase de participación (proyección y anticipación local) y el mecanismo de transición entre ambos aportando información sobre cómo se generan los procesos de abstracción de la idea de múltiplo común durante la resolución del problema.

Los resultados obtenidos indican que, cuando los estudiantes no reconocían la idea de mínimo común múltiplo como instrumento para la resolución del problema, algunos de ellos generaban un proceso de resolución en el que se podía identificar el uso de sus conocimientos previos sobre la estructura multiplicativa de los naturales y, en algunos casos, la construcción de la idea de múltiplo común y mínimo común múltiplo a través de la coordinación de la información.

La manera en la que se ha manifestado el uso de los conocimientos previos ha sido mediante la generación de un conjunto de casos particulares y la realización de una serie de acciones sobre ellos o la interpretación cualitativa de manera global de la situación. Por una parte, el uso de casos particulares es un intento de obtener información general sobre la situación a partir de lo que ya se conoce. Por otra parte, están los estudiantes a los que la interpretación cualitativa global de la situación les permite identificar relaciones relevantes que les ayudan a generar la búsqueda guiada que ha caracterizado el proceso de coordinación de la información. Las dos situaciones descritas concuerdan con el fenómeno «reducción de la abstracción» identificado por Hazzan y Zazkis (2005). Desde esta perspectiva algunos procesos mentales de los estudiantes pueden ser atribuidos a una tendencia a convertir una idea poco familiar (en este caso, la situación planteada por el problema) en algo más familiar (en este caso, la concreción de la situación en ciertos casos particulares).

Para los estudiantes que generaban casos particulares, esto les permitió llevar a cabo comparaciones entre distintos múltiplos de cada uno de los lados de la baldosa buscando un valor que coincida, es decir, buscando un múltiplo común. Esta búsqueda lleva a clasificar mentalmente los casos particulares y a establecer relaciones entre ellos. Sin embargo, los datos ponen de manifiesto que la simple generación de casos particulares no es suficiente para construir la idea de múltiplo común. Hemos mostrado protocolos en los que se realizaba una búsqueda de casos particulares sin llegar a obtener información general sobre la situación problemática (momento de proyección) y casos en los que la búsqueda inicial se transformaba en una búsqueda estructurada que permitía aproximarse a la obtención de un múltiplo común (momento de anticipación local). La forma en que los estudiantes coordinaban la información procedente de los casos particulares marcó la diferencia entre un comportamiento y otro.

El momento de proyección se caracteriza por la generación de información y la incapacidad para ver dicha información desde una perspectiva diferente que le permitiera focalizarse en la estructura de los casos particulares. Así, 
en este primer momento los estudiantes comparaban casos particulares que respondían a la relación $33 \cdot a=30 \cdot a$, colocando el mismo número de baldosas a cada lado de la pista y concluyendo que es imposible construir una pista cuadrada con baldosas de este tipo. En este sentido, los estudiantes en la fase de proyección se centran en el proceso de multiplicar más que en la estructura formada por los dos conjuntos de múltiplos generados. Esta característica se recoge en la columna izquierda de la figura 5.

Para que se inicie la transición del momento de proyección al momento de anticipación local, es necesario que el estudiante cambie el foco de atención al observar el conjunto de casos particulares para obtener información sobre la estructura general del conjunto de datos. Este cambio de foco concuerda con el identificado por Dörfler (2002) y parece ser el desencadenante de la coordinación de la información que permite identificar ciertas relaciones que son usadas para estructurar la búsqueda guiada de nuevos casos particulares.

Figura 5

Proceso de abstracción durante la resolución del problema.

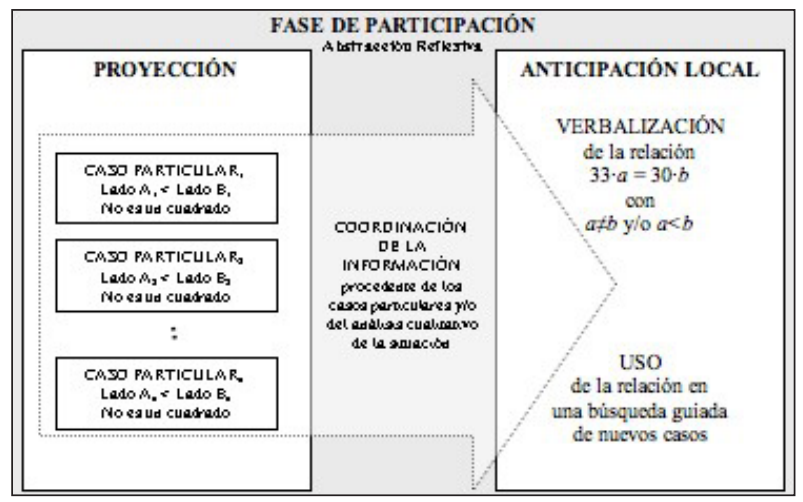

La búsqueda guiada que caracteriza el momento de anticipación local de la fase de participación sólo se produce si hay una coordinación adecuada de información (Figura 5) que permite estructurar las acciones que se realizan. La coordinación de la información permite hacer emerger la idea de múltiplo común durante la resolución de problemas y caracteriza la transición entre el momento de proyección y el momento de anticipación local como una manifestación del proceso de abstracción reflexiva. Lo que nuestra investigación ha mostrado es que la forma en que los estudiantes usan los casos particulares y la interpretación cualitativa de las relaciones multiplicativas entre las cantidades en la situación para obtener información determina su proceso de construcción de la idea de múltiplo.

Esta descripción de los procesos seguidos por los estudiantes permite identificar algunos aspectos comunes a lo descrito por Cañadas, Deulofeu, Figueiras, Reid y Yevdokimov (2008) en lo que se refiere a los pasos para la inducción empírica a partir de un número finito de casos. Sin embargo, nuestro problema es de naturaleza distinta a los problemas de generalización de patrones numéri- cos. La solución del problema no consiste en identificar un patrón, realizar una conjetura y justificarla (Cañadas et al., 2008), sino en identificar una estructura general que permita encontrar un caso particular que cumpla las condiciones del problema. Esto nos ha permitido establecer diferentes momentos dentro del proceso de abstracción del concepto mínimo común múltiplo.

Otro resultado aportado por nuestra investigación es la aplicación de la distinción entre fase de anticipación y fase de participación propuesta por Tzur y Simon (2004) al concepto mínimo común múltiplo. En este sentido, la distinción entre fases nos ha permitido distinguir diferentes comportamientos de los estudiantes al resolver problemas destacando el bajo porcentaje de estudiantes $(21,1 \%)$ que mostraron una comprensión del concepto de mínimo común múltiplo que les permita evocarlo y utilizarlo para resolver el problema. Sin embargo, el hecho de que tan pocos estudiantes de 15-16 años reconocieran el mínimo común múltiplo como estructura de la situación aporta nuevas evidencias de la dicotomía identificada por Brown y otros (2002) entre el método de la descomposición factorial y la comprensión del concepto mínimo común múltiplo de dos o más números. Estos autores consideran que el algoritmo no se construye a través de una aplicación consciente de la comprensión previa de la estructura multiplicativa, sino como un procedimiento memorizado que asocian al concepto mínimo común múltiplo. No es de extrañar, por tanto, que los estudiantes no reconozcan su utilidad en nuestra situación problemática, pues asocian la idea de mínimo común múltiplo a un procedimiento y no a su significado, lo cual según Monaghan y Ozmatar (2006) sería un indicador de la no consolidación del concepto.

Los resultados de esta investigación aportan información sobre la construcción del significado de la idea de múltiplo común como parte del conocimiento de los estudiantes del esquema de divisibilidad y la estructura multiplicativa en el dominio de los naturales. Si consideramos el aprendizaje de un nuevo concepto matemático como un proceso que conlleva la construcción de nuevas estructuras cognitivas a partir de las estructuras previamente disponibles, es necesario proporcionar a los estudiantes experiencias que les permitan modificar dichas estructuras. Según Steffe (1994) el diseño de secuencias de problemas que cumplan esta condición debe estar basado en un conocimiento detallado de los conocimientos que inicialmente son accesibles para el estudiante así como de los mecanismos cognitivos asociados a la construcción de los nuevos conceptos. De esta forma, los resultados de nuestra investigación pueden ser usados como base para el diseño de secuencias de enseñanza en futuras investigaciones que sirvan para formular nuevos modos de proceder para los profesores. En este sentido son necesarias nuevas investigaciones que amplíen el campo de problemas propuestos y que centren la atención en la relación entre el algoritmo de cálculo del mínimo común múltiplo basado en la descomposición factorial de los números y el significado de la idea de múltiplo. Estas nuevas investigaciones deberían aportar información y complementar nuestro conocimiento sobre cómo los estudiantes de educación secundaria llegan a comprender la idea de múltiplo común de varios números como un elemento integrante del esquema de la divisibilidad en el dominio de los números naturales. 


\section{REFERENCIAS BIBLIOGRÁFICAS}

BODÍ, S. (2006). Análisis de la comprensión de divisibilidad en el conjunto de los números naturales. Tesis doctoral. Departamento de Innovación y Formación Didáctica. Universidad de Alicante <http://hdl.handle.net/10045/7757>.

BODÍ, S., VALLS, J. y LLINARES, S. (2005). El análisis del desarrollo del esquema de divisibilidad en N. La construcción de un instrumento. NÚMEROS, 60, pp. 3-24.

BODÍ, S., VALLS, J. y LLINARES, S. (2007). La comprensión de la divisibilidad en $\mathrm{N}$ : un análisis implicativo. En Gras, R. et al. (eds.). Nouveaux Apports Théoriques à l'Analyse Statistique Implicative et Applications: 4èmes Rencontres Internationales d'Analyse Statistique Implicative, pp. 99-110. Castellón: España <http://hdl.handle.net/10045/8015>.

BROWN, A. (2002). Patterns of thought and prime factorization. En Campbell, S. y Zazkis, R. (eds.). Learning and Teaching Number Theory, pp. 131-137. Westport: Ablex Publishing.

BROWN, A., THOMAS, K. y TOLIAS, G. (2002). Conceptions of divisibility: Success and understanding. En S. Campbell y R. Zazkis (eds.). Learning and Teaching Number Theory, pp. 83-95. Westport: Ablex Publishing.

CAMPBELL, S.R. y ZAZKIS, R. (Eds.) (2002). Learning and teaching number theory: Research in cognition and instruction. Westport, Conn.: Ablex Pub.

CAÑADAS, M.C., DEULOFEU, J., FIGUEIRAS, L., REID, D.A. y YEVDOKIMOV, O. (2008). Perspectivas teóricas en el proceso de elaboración de conjeturas e implicaciones para la práctica: tipos y pasos. Enseñanza de las Ciencias, 26(3), pp. 431-444.

CLEMENT, J. (2000). Analysis of clinical interviews: Foundations and model viability, en Kelly, A.E. y Lesh, R.A. (eds.). Handbook of Research Design in Mathematics and Science Education, pp. 547-590. Mahwah, NJ: Lawrence Erlbaum Associates, Pbs.

DÖRFLER, W. (2002). Formation of mathematical objects as decision making. Mathematical Thinking and Learning, 4(4), pp. 337-350.

DUBINSKY, E. (1991). Reflective abstraction in advanced mathematical thinking, en Tall, D. (ed.). Advanced Mathematical Thinking, pp. 95-123. Dordrecht: Kluwer Academic Publishers.

EMPSON, B., JUNK, D., DOMÍNGUEZ, H. y TURNER, E. (2005). Fractions and the coordination of multiplicatively related quantities: A cross-sectional study of children's thinking. Educational Studies in Mathematics, 63, pp. 1-28.

EMPSON, S.B. y TURNER, E (2006). The emergence of multiplicative thinking in children's solution to paper folding tasks. Journal of Mathematical Behavior, 25, pp. 46-56.

FREUDENTHAL, H. (1983). Didactical phenomenology of mathematical structures. Dordrecht: Reidel.

GREER, B. (1994). Extending the meaning of multiplication and division, en Harel, G. y Confrey, J. (eds.). The Development of Multiplicative Reasoning in the Learning of Mathematics, pp. 61-85. Albany: NY: SUNNY Press.
HAREL, G. y CONFREY, J. (Eds.) (1994). The development of multiplicative reasoning in the learning of mathematics. Albany, NY: SUNNY Press.

HAZZAN, O. y ZAZKIS, R. (2005). Reducing abstraction: the case of school mathematics. Educational Studies in Mathematics, 58(1), pp. 101-119.

LLINARES, S. y ROIG, A.I. (2008). Secondary School students' construction and use of mathematical models in solving word problems. International Journal of Science and Mathematics Education, 6, pp. 505-532.

MONAGHAN, J. y OZMANTAR, F. (2006). Abstraction and consolidation. Educational Studies in Mathematics, 62(3), pp. 233-258.

PIAGET, J. (2001). Studies in reflecting abstraction. Sussex: Psychology Press (Editado en 1977 por Presses Universitaires de France).

RAMFUL, A. y OLIVE, J. (2008). Reversibility of thought: An instance in multiplicative tasks. The Journal of Mathematical Behavior, 27, pp. 138-151.

ROIG, A.I. (2008). Análisis de las fases del proceso de abstracción matemática en estudiantes de secundaria. Tesis doctoral inédita. Departamento de Innovación y Formación Didáctica. Universidad de Alicante.

SIERRA, M., GONZÁLEZ, M.T., GARCÍA, A. y GONZÁLEZ, M. (1989). Divisibilidad. Madrid: Síntesis.

SIMON, M. A., TZUR, R., HEINZ, K. y KINZEL, M. (2004). Explicating a mechanism for conceptual learning: Elaborating the construct of reflective abstraction. Journal for Research in Mathematics Education, 35(5), pp. 305-329.

SRIRAMAN, B. (2004). Reflective abstraction, uniframes and the formulations of generalizations. Journal of Mathematical Behavior, 23, pp. 205-222.

STEFFE, L. (1994). Children's multiplying schemes. En Harel, G. y Confrey, J. (eds.). The Development of Multiplicative Reasoning in the Learning of Mathematics, pp. 3-40. Albany, NY: State University of New York Press.

TZUR, R. (2003). Teacher and students' joint production of a reversible fraction conception. En Pateman, N., Dougherty, B. y Zilliox, J. (eds.). Proceedings of the $27^{\text {th }}$ Conference for the International Group for the Psychology of Mathematics Education, 4, pp. 315-322. Honolulu: PME.

TZUR, R. (2004). Teacher and students' joint production of a reversible fraction conception. Journal of Mathematical Behavior, 23(1), pp. 93-114.

TZUR, R. (2007). Fine grain assessment of students' mathematical understanding: participatory and anticipatory stages in learning a new mathematical concept. Educational Studies in Mathematics, 66, pp. 273-291.

TZUR, R. y SIMON, M.A. (2004). Distinguishing two stages of mathematics conceptual learning. International Journal of Science and Mathematics Education, 2, pp. 287-304.

VERGNAUD, G. (1996). The theory of conceptual fields. En 
L.P. Steffe, P. Nesher, P., Cobb, G.A. Goldin y B. Greer (eds.). Theories of Mathematical Learning, pp. 219-239. Hillsdale, NJ: Lawrence Erlbaum.

VERSCHAFFEL, L., GREER, B. y TORBEYNS, J. (2006). Numerical thinking, en Gutiérrez, A. y Boero, P. (eds.). Handbook of Research on the Psychology of mathematics Education, pp. 51-82. Rotterdam/Taipei: Sense Publishers.

ZAZKIS, R. (1998). Divisors and quotients: Acknowledging polysemy. For the Learning of Mathematics, 18(3), pp. 27-30.

ZAZKIS, R. (2000). Factors, divisors and multiples: Exploring the web of students' connections. En Schoenfeld, A., Kaput, J. y Dubinsky, E. (eds.). Vol. 4. Research in Collegiate Mathematics Education, pp. 210-238. Providence, RI: American Mathematical Society.

ZAZKIS, R. (2008). Divisibility and transparency of number representations. En Carlson, M.P. y Rasmussen, C. (eds.).
Making the Connection: Research and Practice in Undergraduate Mathematics, pp. 81-92. MAA notes.

ZAZKIS, R. y CAMPBELL, S. (1996). Divisibility and multiplicative structure of natural numbers: preservice teachers' understanding. Journal for Research in Mathematics Education, 27(5), pp. 540-563.

ZAZKIS, R. y CAMPBELL, S.R. (Eds.) (2006). Number Theory in Mathematics Education: Perspectives and Prospects. Mahwah, NJ: Lawrence Erlbaum Press.

ZAZKIS, R. y GADOWSKY, K. (2001). Attending to transparent features of opaque representations of natural numbers. En Couco, A. (ed.). NCTM 2001 Yearbook: The Roles of Representation in School Mathematics, pp. 41-52. Reston, VA: NCTM.

ZAZKIS, R. y LILJEDAHL, P. (2004). Understanding primes: The role of representation. Journal for Research in Mathematics Education, 35(3), pp. 164-186. 


\title{
Construction of the common multiple concept in the domain of whorle numbers
}

\author{
Roig, Ana Isabel, Llinares, Salvador y Penalva, María del Carmen \\ Departamento de Innovación y Formación Didáctica. Universidad de Alicante \\ a.roig@ua.es \\ sllinares@ua.es \\ carmina.penalva@ua.es
}

\section{Summary}

Understanding the concepts of divisor and multiple, and the relations "to be a divisor of" and "to be a multiple of", is an important aspect of the divisibility scheme of whole numbers. Taking into account this fact, research has focused on characterizing this understanding and determining its development. In this context, the aim of this paper is to clarify some aspects of the construction process that takes place when Secondary School students solve a word problem related with the concepts of common multiple and lowest common multiple.

From a piagetian point of view, the development of a new concept is a process that entails the construction of new cognitive structures from previously available structures. The mechanism that carries out this construction is the Reflexive Abstraction (Piaget, 2001). A theoretical approach that characterizes this mechanism is the Reflexion on Activity-Effect Relationship proposed by Simon and colleagues (Simon, Tzur, Heinz y Kinzel, 2004). They claim that the abstraction from a set of activity-effect relationships allows the learner to anticipate the effects of new activities without carrying them out. In this process, Tzur \& Simon (2004) distinguish two stages, a Participatory Stage and an Anticipatory Stage. In the Participatory Stage, the learner sorts the activity-effect records out, compares, relates, searches and coordinates information to identify a regularity. Then, the information is used to anticipate the effects of new activities without carrying them out. But this knowledge is only available in the context of the activity through which it has been developed. In the Anticipatory Stage, the learner is able to recognise the involvement of the conception (the regularity in the set of activity-effect relationships) and to use it independently from the context or problem situation. From this theoretical framework, the purpose of this research is to provide information about how Secondary School students generate the abstraction of common multiple and lowest common multiple concepts.

The participants were 71 Secondary School students in the last year of compulsory education (15-16 years old). The data came from two sources: (1) students' written solutions to a word problem which mathematical structure was organized around the idea of common multiple and, (2) the transcriptions of clinical interviews carried out after the written solutions. During the interviews the students were asked to justify their solutions and their decisions. We made a descriptive analysis of the written solutions and the associated interviews. Firstly we used the distinction between Participatory and Anticipatory Stages in order to categorize the different students' approaches in the resolution of the word problem. We took into account the previous knowledge used by the students. This categorization allows us to notice the existence of considerable differences in the Participatory Stage solutions. This situation leads us to a second analysis focused on these solutions. For this second analysis we used the Reflexion on Activity-Effect Relationship in order to identify the students' actions and how these actions were evaluated by them. From this process, we identified two cognitive moments in the constructive process of the Participatory Stage. This distinction focus on how students established relations between different mathematical elements when using particular cases and if they were or were not able to coordinate the information arising from them.

The results show that when the students couldn't recognize the common multiple as an instrument to solve the problem, some of them used their previous knowledge about the multiplicative structure of whole numbers, and in some cases they coordinated information to construct the common multiple and lowest common multiple. The use of previous knowledge has been manifested by the generation and use of a set of particular cases and the realization of a set of actions from them. The use of particular cases is an attempt to obtain general information about the established situation. This allows students to compare different multiples of two numbers looking for a common multiple. With this search students could classify the particular cases by establishing relations between them. Furthermore, students could not obtain general information (projection moment) or could transform the search in a structured one. This last point approximated them to the common multiple (local anticipation moment). The way in which the students coordinated the information provided by the particular cases was the difference between these behaviours. 
